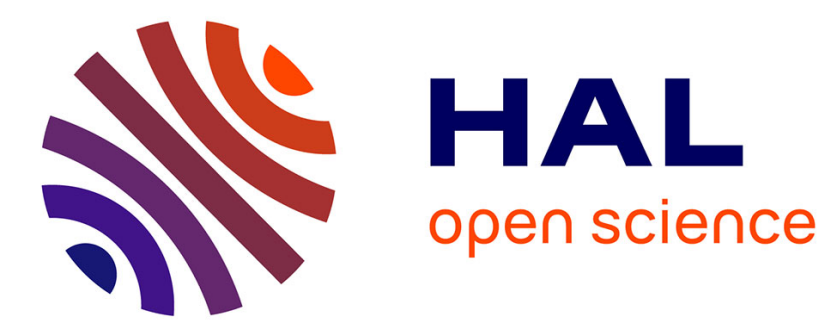

\title{
Using a cw 465nm laser to probe metastable He2 molecules
}

Geneviève Tastevin, Bartosz Glowacz, Pierre-Jean Nacher

\section{To cite this version:}

Geneviève Tastevin, Bartosz Glowacz, Pierre-Jean Nacher. Using a cw 465nm laser to probe metastable He2 molecules. Journal of Low Temperature Physics, 2009, pp.Online first. 10.1007/s10909-009-0050-5 . hal-00433545

\section{HAL Id: hal-00433545 \\ https://hal.science/hal-00433545}

Submitted on 19 Nov 2009

HAL is a multi-disciplinary open access archive for the deposit and dissemination of scientific research documents, whether they are published or not. The documents may come from teaching and research institutions in France or abroad, or from public or private research centers.
L'archive ouverte pluridisciplinaire HAL, est destinée au dépôt et à la diffusion de documents scientifiques de niveau recherche, publiés ou non, émanant des établissements d'enseignement et de recherche français ou étrangers, des laboratoires publics ou privés. 


\title{
Using a cw 465nm laser to probe metastable $\mathrm{He}_{2}$ molecules
}

\author{
G. Tastevin, ${ }^{1}$ B. Glowacz, ${ }^{1,2}$ and P.-J. Nacher ${ }^{1}$ \\ ${ }^{1}$ Laboratoire Kastler Brossel, Ecole Normale Supérieure; CNRS; UPMC; 24 rue Lhomond, F75005 Paris, France \\ ${ }^{2}$ Atomic Optics Department, Jagiellonian University - Ul. Reymonta 4, 30-059 Kraków, Poland
}

\begin{abstract}
A cw $465 \mathrm{~nm}$ laser has been built for sensitive detection of helium metastable molecules by resonant absorption on the $a^{3} \Sigma_{u}^{+}(v=0)-e^{3} \Pi_{g}(v=0)$ transition band. The frequency-mixing radiation is obtained from commercial laser diodes in a periodically-poled KTP non-linear crystal. The 1.3-nm tuning range includes the main rotational lines of ${ }^{3} \mathrm{He}_{2},{ }^{4} \mathrm{He}_{2}$, and ${ }^{3} \mathrm{He}-{ }^{4} \mathrm{He}$ dimers. Measurements of absolute molecular densities down to a few $10^{9} \mathrm{~cm}^{-3}$ are reported in low pressure $(1-400 \mathrm{mbar})$ room temperature He gas excited by a weak rf discharge. Unsophisticated detection techniques provide signals with good signal-to-noise ratios thanks to the narrow absorption linewidths (a few $\mathrm{GHz}$, due to Doppler and moderate collisional broadenings) in the fully resolved spectrum. Prospects for use or upgrade of this blue laser to probe the broadened and shifted molecular lines in condensed He are discussed.
\end{abstract}

PACS numbers: 33.20.Kf, 42.65.Ky, 67.25.-k

Keywords: $\mathrm{He}_{2}$ metastable molecules, laser absorption, frequency mixing in PP-KTP, spectroscopy in dense $\mathrm{He}$

\section{INTRODUCTION}

Metastable molecules $\left(\mathrm{He}_{2}^{*}\right)$ are ubiquitous in helium systems. They are produced by any excitation process that feeds in enough energy to create excited, ionic or metastable atomic species (electric discharge, bombardment by charged particles, irradiation with ionizing radiation or intense laser pulses), provided that the density of ground state atoms is high enough to allow conversion by 3-body collisions. Extensive work has dealt with room temperature gas for basic studies of this unique dimer and of the complex processes involved in He plasmas, with a renewed interest driven by a variety of applications both at low and high pressures. Low temperatures provide an exceptionally clean environment where the lowest-energy triplet state $a^{3} \Sigma_{u}^{+}$is very long-lived both in gas and liquid He [1]. Emission spectra bring a wealth of information about the processes of formation [2] and decay [3] of $\mathrm{He}_{2}^{*}$. However, only absorption techniques can allow straightforward absolute density measurements. They also provide complementary spectroscopic data [4, 5] for quantitative investigation of the structural changes induced in the fluid by the electronic excitation and of the de-excitation channels [3, 6, 7]. Moreover, they potentially provide high sensitivity and time resolution for improved detection, imaging, or tracking of dissolved $\mathrm{He}_{2}^{*}$ (currently performed using light-induced fluorescence techniques $[8,9$ ).

So far, $\mathrm{He}_{2}^{*}$ absorption has been probed at low temperatures with fairly low resolution using broad lamps and monochromators [6, 10] or with sophisticated lasers [11] and systematic studies involve only the most prominent lines 177. Here, a compact laser source designed for absolute measurements of molecular density by laser absorption on the $a^{3} \Sigma_{u}^{+}(v=0)-e^{3} \Pi_{g}(v=0)$ vibrational band at $465 \mathrm{~nm}$ is presented. Detailed description and characterisation will be provided elsewhere [12]. The present report focuses on the sensitivity of absorption measurements demonstrated in low pressure room temperature He gas. Prospects for upgrade of the laser and for use as a tool to probe $\mathrm{He}_{2}^{*}$ in cold and condensed He are discussed.

\section{LASER SET-UP AND PERFORMANCES}

Continuous-wave frequency up-conversion is performed with a commercial $2 \times 1 \times 10 \mathrm{~mm}^{3}$ crystal of periodically poled $\mathrm{KTiOPO}_{4}$ (PP-KTP) (Raicol Crystals Ltd.), selected for optimal performance [13]. The crystal is phase-matched for frequency doubling at $930 \mathrm{~nm}$. Given the prohibitive cost-to-power ratio of a custom made laser diode (LD) at $930 \mathrm{~nm}$ (more than twice higher price per item with only 40-mW output), emission at $465 \mathrm{~nm}$ is actually obtained by

*Published online in the Journal of Low temperature Physics, 2009, DOI: 10.1007/s10909-009-0050-5) http://www.springerlink.com link . asp?id=104917 
frequency mixing from two 100-mW single-mode distributed feedback LDs at 923.6 and $937.6 \mathrm{~nm}$ available off-theshelf. The difference in wavelengths excludes the usual configuration with incident perpendicular beams collinearly combined using a dichroic mirror. The laser set-up (Fig. 1) therefore includes a set of three dispersive glass prisms that introduce a small individual difference in deflection angle $\left(0.005^{\circ} \mathrm{nm}^{-1}\right.$ at minimum deviation $)$ and several mirrors that provide incident beams with a matched small tilt angle.

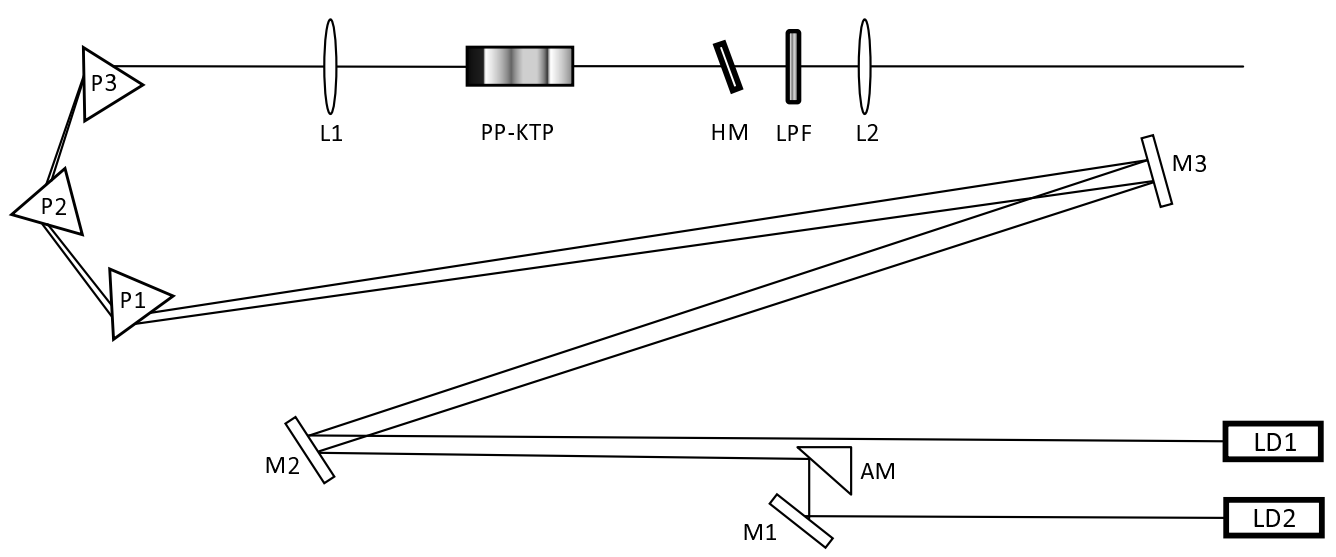

FIG. 1: Laser set-up. LD1, 2: LD at 923.6, $937.6 \mathrm{~nm}$; M: mirror; AM: angle mirror; P: prism; L: lens; HM: hot mirror; LPF: low pass filter. HM and LPF both filter out the $465 \mathrm{~nm}$ radiation.

The power at the output of the crystal reaches $0.23 \mathrm{~mW}$ at $465 \mathrm{~nm}$ for $75 \mathrm{~mW}$ and $80 \mathrm{~mW}$ input at 923 and $937 \mathrm{~nm}$, respectively. The effective conversion efficiency $\left(3.9 \% \mathrm{~W}^{-1} \mathrm{~cm}^{-1}\right)$ is in line with expectations taking into account the elliptical shapes of the collimated LD beams (typically $1 \mathrm{~mm} \times 2 \mathrm{~mm}$ FWHM) [12]. This is slightly lower than the $4.6 \% \mathrm{~W}^{-1} \mathrm{~cm}^{-1}$ maximum computed for perfect Gaussian beams and optimal beam waist [13]. The laser can be tuned over $1.3 \mathrm{~nm}$ at a rate of $39 \mathrm{GHz}{ }^{\circ} \mathrm{C}^{-1}$ by temperature control of the infrared LDs (operating range: $5-45^{\circ} \mathrm{C}$ ). The crystal temperature must be correlatively adjusted between 20 and $68{ }^{\circ} \mathrm{C}$ to preserve optimal phase matching, due to the measured $0.07 \mathrm{~nm}$ FWHM $(97 \mathrm{GHz})$ tolerance at fixed PP-KTP temperature [14]. The laser is very stable in intensity and its spectral bandwidth, set by the input LDs, is on the order of a few $\mathrm{MHz}$.

\section{ABSORPTION MEASUREMENTS}

Molecular absorption on the $a \rightarrow e(0-0)$ transition is probed in sealed glass cells (length $11 \mathrm{~cm}$, inner diameter $1.6 \mathrm{~cm}$ ) filled with He gas at pressures ranging between 8 and 400 mbar, in conditions typical for room temperature optical pumping: excitation by rf discharge at $3 \mathrm{MHz}$ using external wire electrodes, with a density of metastable $2^{3} \mathrm{~S}$ atoms in the $10^{9}-10^{12} \mathrm{~cm}^{-3}$ range (this density is simultaneously measured using the $2^{3} \mathrm{~S}-2^{3} \mathrm{P}$ atomic line at $1083 \mathrm{~nm}$ with a commercial distributed Bragg reflector LD [15, 16]). The single-pass transmitted blue light is monitored by a silicon photodiode.

The measured absorption ranges from a few $10^{-4}$ to $10 \%$, depending on pressure and discharge intensity. This corresponds to a $\mathrm{He}_{2}^{*}$ density in the $10^{8}-10^{12} \mathrm{~cm}^{-3}$ range [17]. Fig. 2 shows a signal recorded with a signal-to-noise ratio (SNR) of order 2000 in pure ${ }^{3} \mathrm{He}$ at 96 mbar. The inset displays the set of rotational lines identified in the accessible part of the ${ }^{3} \mathrm{He}_{2}^{*}$ spectrum [18]. The intensity distribution corresponds to thermal equilibrium around $300 \mathrm{~K}$, with stronger even-numbered lines due to the nuclear spin $1 / 2$ of ${ }^{3} \mathrm{He}$ [18]. The less numerous rotational lines of ${ }^{4} \mathrm{He}_{2}^{*}$ have been similarly probed (data not shown), its band head lying only $0.06 \mathrm{~nm}$ below that of ${ }^{3} \mathrm{He}_{2}^{*} 18$, 19.

Sensitivity easily reaches $10^{-5}$ with a lock-in amplifier when either the gas excitation or the laser output are modulated. Molecular density is here varied by low frequency (a few $\mathrm{Hz}$ ) modulation of the discharge amplitude, the $\mathrm{He}_{2}^{*}$ lifetime being ultimately limited to tens of milliseconds by diffusion to the walls $\left(D=310 \mathrm{~cm}^{2} \mathrm{~s}^{-1}\right.$ for ${ }^{4} \mathrm{He}_{2}^{*}$ at 1 Torr [20]). A double-modulation scheme can further improve the SNR. Signal modulation at significantly higher frequencies can be achieved using, e.g., a mechanical chopper to modulate the laser intensity. A cheaper alternative is to periodically drive one of the input LDs above and below laser threshold by strong modulation of its feeding current. For sharp resolved lines, the modulation of one feeding current may also be adjusted so as to bring the laser on- and off-resonance with $\mathrm{He}_{2}^{*}$ and to obtain a $100 \%$ modulation of the absorption rate with moderate impact on the laser power. 


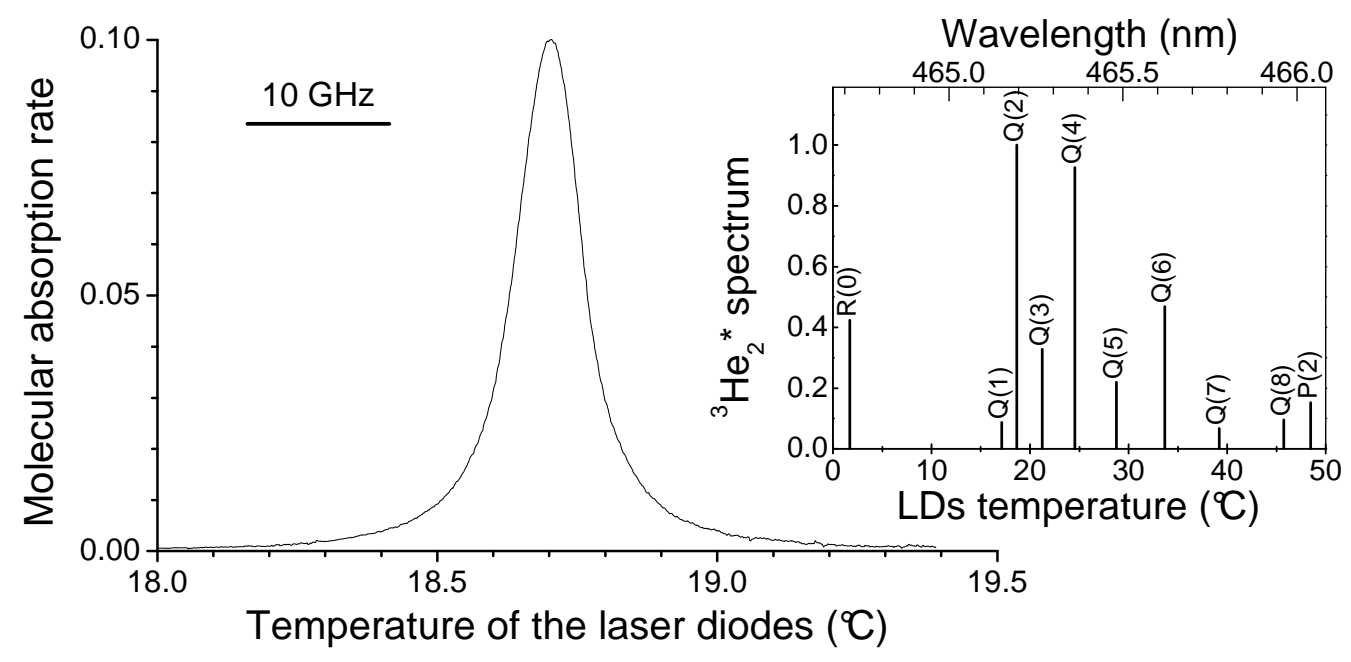

FIG. 2: Resonant laser absorption signal obtained on the $\mathrm{Q}(2)$ line in 96 mbar ${ }^{3} \mathrm{He}$ gas at room temperature. Inset: Observed and identified lines in the ${ }^{3} \mathrm{He}_{2}^{*}$ rotational spectrum. Line intensities have been normalised to the highest peak, $\mathrm{Q}(2)$.

\section{DISCUSSION}

Well resolved rotational lines are observed, with Voigt profiles that result from combined Doppler and collisional broadenings (Gaussian FWHM $W_{D}=3.25 \mathrm{GHz}$ and Lorentzian FWHM $W_{L}=3.72 \mathrm{GHz}$, respectively, for Fig. 2 data). The peak heights can thus easily be related to the absorption rate $A$ and transmission rate $T=1-A$. Their reciprocals uniformly scale linearly with $W_{L}$ at small $W_{L}$ and this increase transiently slows down when $W_{L}$ becomes comparable to the average splitting, due the rising contribution of the neighbouring lines. For instance, the $\mathrm{Q}(2)$ peak in Fig. 2 is already 2.3 times smaller than it would be at $W_{L}=0$, and could be about 40 (resp. 64) times smaller for $W_{L}=100$ (resp. 200) GHz. The $\mathrm{He}_{2}^{*}$ number density $N_{m}$ can be directly inferred from the measured optical density using the expression $-\ln T=N_{m} \sigma l$, where $l$ is the optical path length and $\sigma$ is the optical cross section. The cross section $\sigma$ lies in the $3-510^{-15} \mathrm{~cm}^{2}$ range for the main ${ }^{4} \mathrm{He}_{2}^{*}$ rotational lines at $465 \mathrm{~nm}$ [17. This value is 100 times lower that of the atomic $1083 \mathrm{~nm}$ line [15, 16].

The 1.3-nm tuning range of the laser includes the main rotational lines of ${ }^{3} \mathrm{He}_{2}$, of ${ }^{4} \mathrm{He}_{2}$, and hence of ${ }^{3} \mathrm{He}$ ${ }^{4} \mathrm{He}$ dimers. It thus allows systematic studies of the $a \rightarrow e$ line that are useful for quantitative comparison with quantum chemistry computations and fundamental to understanding of many collisional and quenching processes. This provides, in particular, accurate tests of the interaction potentials between ground and excited states, especially relevant for investigations on low temperature systems such as quantum He nanodroplets and laser-cooled metastable He [21].

Finding a laser with suitable wavelength and characteristics is often difficult. Today, established up- and downfrequency conversion methods and high quality crystals released in the last 15 years allow experimentalists to build lasers tailored to their needs. Moreover, the limited time and effort required to put together an appropriate system is usually worth the cost difference between the set of optical components and a custom-made source. The cw $465 \mathrm{~nm}$ laser described in this report has been specifically developed for $\mathrm{He}_{2}^{*}$ absorption measurements that require very little power and narrow spectral bandwidth. Its innovative design is relevant when available lasers have wavelengths close to, but not exactly at, twice the desired one. Various strategies exist for a straightforward increase of the laser performances. For higher laser output, one can use a ring or standing-wave enhancement cavity to boost up the conversion efficiency [13] and use tapered amplifiers [22] or other $930 \mathrm{~nm}$ lasers [13] to increase the input power. Alternatively, $465 \mathrm{~nm}$ light can be obtained by frequency mixing from very different wavelengths combining, e.g., a diode-pumped solid-state laser for high input power and a small power LD to retain the tuning and high-frequency modulation abilities [23]. When optical saturation of the entire line profile is required, the LD can also be replaced by a diode array (nm-broad) or a fiber laser (bandwidths range from tens of $\mathrm{kHz}$ to nanometer) to have more output power and to optimally match the absorption linewidth, taking advantage of the wide phase-matching bandwidth for sum-frequency generation in PP-KTP 24, 25]. Of course, different input wavelengths and PP-KTP grating period (or angle tuning) can be selected to probe other visible $\mathrm{He}_{2}^{*}$ or impurity lines.

Following early studies of low pressure microwave gas discharges and applications in microelectronics, recent $\mathrm{He}$ work strongly focuses on high pressure plasmas (with or without selected impurities) for use in surface treatment, 
laser sources, plasma displays, and biology. With room temperature $\mathrm{He}_{2}^{*}$ densities up to $10^{15} \mathrm{~cm}^{-3}$ [17, 26, 27, 28, a compact $465 \mathrm{~nm}$ laser may be a useful high resolution dedicated probe for plasma diagnosis or study of rotational relaxation and collisional transfer rates 27, 29, providing higher sensitivity and resolution than He or Xe excitation lamps 20, 27] and tunable dye lasers [17, 30. The laser can also be used for new investigations in cold He gas where unusual spectral features, differences between isotopomers, and specific molecular dynamics are observed 10 , 31, 32. Sensitive measurements are expected thanks to the still fairly high $\mathrm{He}_{2}^{*}$ densities $\left(10^{12} \mathrm{~cm}^{-3}\right.$ at $\left.4.2 \mathrm{~K}\right)$ and to the nicely resolved absorption lines [10, 33, 34. For light-induced fluorescence studies, one advantage of the $465 \mathrm{~nm}$ line potentially lies in the proximity between the $e, d$, and $f$ states of $\mathrm{He}_{2}^{*}[17$.

In its current configuration, the laser may also be used to probe $\mathrm{He}_{2}^{*}$ with high spectral and temporal resolution in He clusters or nanodroplets and to get insight on their dynamics inside the fluid. In both cases, sharp and unshifted lines with well resolved rotational structures have been observed, due to the presence of free molecules expelled from the surface [35, 36]. Direct observation is potentially more challenging for $\mathrm{He}_{2}^{*}$ floating at the surface of superfluid He [37] or dissolved in bulk liquid, despite the very long lifetimes (up to $13 \mathrm{~s}$ [1]) and high densities $\left(10^{12}-10^{14} \mathrm{~cm}^{-3}\right.$ ) reported in this latter system. Significant line shifts and broadenings result from the repulsive interaction between $\mathrm{He}_{2}^{*}$ and the surrounding liquid, leading to partly resolved rotational spectra [4, 5]. A good quantitative description of both systems is required to account for the non trivial observed changes, that strongly depend on initial and final electronic states [6, 7]. Emission and absorption lines shift at markedly different rates with increasing pressure [4, 5], in a way that also differs from that observed in He gas [38]. If most lines experience a blue shift on the order of several tenths of nanometer per bar, the triplet $a \rightarrow b$ absorption line, for instance, does not shift at all [5], 6]. To our best knowledge, no computations and very few data are available for the $a \rightarrow e(0-0)$ line at $465 \mathrm{~nm}$. This line is weak but clearly present in fluorescence spectra of liquid He at saturated vapour pressure [3] and appears quite sharp in high-resolution $4.2 \mathrm{~K}$ studies [39, 40]. So far, no significant shift has been measured for light emission induced by a corona discharge in liquid ${ }^{4} \mathrm{He}(464.95 \pm 0.10 \mathrm{~nm}$ [40]).

Recently, visualisation of $\mathrm{He}_{2}^{*}$ clouds and measurements of normal-fluid velocity in superfluid He using optically tagged molecules have been demonstrated [9]. The detection scheme involves 2-photon excitations of $a \rightarrow d$ transitions and monitoring of the $d \rightarrow c$ spontaneous emission. This scheme has also be proposed for applications such as detection of ionizing radiation [41] and imaging of Compton gamma rays [11]. In addition to the two pulsed lasers used for pump and probe excitation, two powerful infrared lasers are required to recover the molecules that end up in off-resonant vibrational levels of the $a^{3} \Sigma_{u}^{+}$state. The intrinsic loss of sensitivity introduced by the fluorescence detection (1\% solid angle) is also partly compensated by the number of cycles each molecule can make during its lifetime (10 - 100 emitted photons, ultimately limited by the $b$ state decay). Detection by absorption on a single resonant dipolar transition potentially provides a high sensitivity without need of high laser power, sophisticated photodetectors, or large optical access inside the cryostat. The main advantage of this technique is to allow absolute density measurements.

[1] D. N. McKinsey, C. R. Brome, J. S. Butterworth, S. N. Dzhosyuk, P. R. Huffman, C. E. H. Mattoni, J. M. Doyle, R. Golub, and K. Habicht. Radiative decay of the metastable $\mathrm{He}_{2}\left(a^{3} \Sigma_{u}^{+}\right)$molecule in liquid helium. Physical Review A, 59(1):200-204, Jan 1999.

[2] D. W. Tokaryk, G. R. Wagner, R. L. Brooks, and J. L. Hunt. Infrared emission spectra from cryogenic proton-irradiated helium gas. The Journal of Chemical Physics, 103(24):10439-10444, 1995.

[3] A. V. Benderskii, R. Zadoyan, N. Schwentner, and V. A. Apkarian. Photodynamics in superfluid helium: Femtosecond laser-induced ionization, charge recombination, and preparation of molecular Rydberg states. The Journal of Chemical Physics, 110(3):1542-1557, 1999.

[4] J. C. Hill, O. Heybey, and G. K. Walters. Evidence of metastable atomic and molecular bubble states in electron-bombarded superfluid liquid helium. Physical Review Letters, 26(20):1213-1216, May 1971.

[5] F. J. Soley and W. A. Fitzsimmons. Pressure shifts and quenching of atomic and molecular states produced in electronbombarded liquid helium. Physical Review Letters, 32(18):988-991, May 1974.

[6] S. Kafanov, A. Parshin, and I. Todoshchenko. Structure and dynamics of the He. Journal of Experimental and Theoretical Physics, 91(5):991-999, November 2000.

[7] J. Eloranta, N. Schwentner, and V. A. Apkarian. Structure and energetics of $\mathrm{He}_{2}{ }^{*}$ bubble-states in superfluid ${ }^{4} \mathrm{He}$. The Journal of Chemical Physics, 116(10):4039-4053, 2002.

[8] A. V. Benderskii, J. Eloranta, R. Zadoyan, and V. A. Apkarian. A direct interrogation of superfluidity on molecular scales. The Journal of Chemical Physics, 117(3):1201-1213, 2002.

[9] W. Guo, J. D. Wright, S. B. Cahn, J. A. Nikkel, and D. N. McKinsey. Metastable helium molecules as tracers in superfluid ${ }^{4}$ He. Physical Review Letters, 102(23):235301+, 2009.

[10] R. L. Brooks, J. L. Hunt, and D. W. Tokaryk. Absorption spectra from high vibrational levels of He2. The Journal of Chemical Physics, 91(12):7408-7414, 1989.

[11] W. G. Rellergert, S. B. Cahn, A. Garvan, J. C. Hanson, W. H. Lippincott, J. A. Nikkel, and D. N. McKinsey. Detection 
and imaging of $\mathrm{He}_{2}$ molecules in superfluid helium. Physical Review Letters, 100(2):025301+, 2008.

[12] Glowacz B., Tastevin G., Nacher P.-J., and Dohnalik T. in preparation. 2010.

[13] R. Le Targat, J. Zondy, and P. Lemonde. 75\%-efficiency blue generation from an intracavity PPKTP frequency doubler. Optics Communications, 247(4-6):471-481, March 2005.

[14] W. P. Risk. Phase-matching characteristics of periodically poled KTP. In M. C. Gupta, W. J. Kozlovsky, \& D. C. MacPherson, editor, Society of Photo-Optical Instrumentation Engineers (SPIE) Conference Series, volume 2700 of Presented at the Society of Photo-Optical Instrumentation Engineers (SPIE) Conference, pages 78-84, May 1996.

[15] E. Courtade, F. Marion, P. J. Nacher, G. Tastevin, K. Kiersnowski, and T. Dohnalik. Magnetic field effects on the 1083 nm atomic line of helium. The European Physical Journal D, 21(1):25-55, October 2002.

[16] M. W. Millard, P. P. Yaney, B. N. Ganguly, and C. A. DeJoseph. Diode laser absorption measurements of metastable helium in glow discharges. Plasma Sources Science Technology, 7:389-394, August 1998.

[17] S. Neeser, R. Tietz, M. Schulz, and H. Langhoff. Lifetime of the $\mathrm{He}_{2}(e), \mathrm{He}_{2}(d)$ and $\mathrm{He}_{2}(f)$ states. Zeitschrift fur Physik D Atoms Molecules Clusters, 31:61-65, March 1994.

[18] G. H. Dieke and E. S. Robinson. The molecular spectrum of He ${ }^{3}$. Physical Review, 80:1-5, October 1950.

[19] C. M. Brown and M. L. Ginter. Spectrum and structure of the $\mathrm{He}_{2}$ molecule VI. Characterization of the states associated with the UAO's 3p $\pi$ and 2s. Journal of Molecular Spectroscopy, 40:302-316, November 1971.

[20] A. V. Phelps. Absorption studies of helium metastable atoms and molecules. Physical Review, 99:1307-1313, August 1955.

[21] D. Vrinceanu and H. R. Sadeghpour. $\mathrm{He}\left(1^{1} \mathrm{~S}\right)-\mathrm{He}\left(2^{3} \mathrm{~S}\right)$ collision and radiative transition at low temperatures. Phys. Rev. A, 65(6), June 2002

[22] R. G. Batchko, M. M. Fejer, R. L. Byer, D. Woll, R. Wallenstein, V. Y. Shur, and L. Erman. Continuous-wave quasiphase-matched generation of $60 \mathrm{mw}$ at $465 \mathrm{~nm}$ by single-pass frequency doubling of a laser diode in backswitch-poled lithium niobate. Optics Letters, 24:1293-1295, September 1999.

[23] S. Johansson, S. Spiekermann, S. Wang, V. Pasiskevicius, F. Laurell, and K. Ekvall. Generation of turquoise light by sum frequency mixing of a diode-pumped solid-state laser and a laser diode in periodically poled KTP. Optics Express, 12, July 2004 .

[24] W. P. Risk and W. Lenth. Diode laser pumped blue-light source based on intracavity sum frequency generation. Applied Physics Letters, 54:789-791, February 1989.

[25] H. Sakata, Y. Furukawa, and T. Yagi. Type II quasi-phase-matched sum-frequency mixing of diode lasers in KTiOPO4 with broad spectral and temperature acceptance bandwidths. Appl. Opt., 43:4922-4928, September 2004.

[26] G. Myers and A. J. Cunningham. Rate measurements of reactions of helium metastable species at atmospheric pressures. II - $\mathrm{He}_{2}\left(2{ }^{3} \Sigma_{u}^{+}\right)$in pure afterglows. J. Chem. Phys., 67:1942-1947, September 1977.

[27] S. Takao, M. Kogoma, T. Oka, M. Imamura, and S. Arai. Optical absorption spectra and kinetic behavior of helium excited diatomic molecule $\left(\mathrm{a}^{3} \Sigma_{u}^{+}\right)$. J. Chem. Phys., 73:148-155, July 1980.

[28] F. Emmert, H. H. Angermann, R. Dux, and H. Langhoff. Reaction kinetics of the $\mathrm{He}(2 \mathrm{P})$ and the $\mathrm{He}_{2} *(\mathrm{a}, \mathrm{v})$ states in high-density helium. Journal of Physics D Applied Physics, 21:667-674, May 1988.

[29] R. Nakata, T. Matsushita, and K. Fukuda. Collisonal electronic and rotational relaxation of molecular helium in an afterglow of pulsed discharge. Japanese Journal of Applied Physics, 16, June 1977.

[30] C. B. Collins and B. W. Johnson. Study of excitation transfer in a flowing helium afterglow pumped with a tuneable dye laser. II. Measurement of the rate coefficient for the rotational relaxation of $\operatorname{He}_{2}\left(3 \mathrm{p}^{3} \Pi_{g}\right)$. J. Chem. Phys., 57:5317-5321, December 1972.

[31] K. Kimura, M. Kataoka, T. Azuma, Y. Ito, Y. Tabata, and Y. Aoki. New emission bands at high-density excitation of dense gas and liquid of helium irradiated by nitrogen ions. Physics Letters A, 133:91-94, October 1988.

[32] Y. Noma, J. H. Choi, T. Tomai, and K. Terashima. Gas-temperature-dependent generation of cryoplasma jet under atmospheric pressure. Applied Physics Letters, 93(10), September 2008.

[33] W. S. Dennis, E. Durbin, W. A. Fitzsimmons, O. Heybey, and G. K. Walters. Spectroscopic identification of excited atomic and molecular states in electron-bombarded liquid helium. Physical Review Letters, 23:1083-1086, November 1969.

[34] R. L. Brooks and J. L. Hunt. Helium emission spectra from doped samples of solid hydrogen and deuterium. J. Chem. Phys., 88:7267-7272, June 1988.

[35] K. Von Haeften, T. Laarmann, H. Wabnitz, and T. Möller. Bubble Formation and Decay in ${ }^{3}$ He and ${ }^{4}$ He Clusters. Physical Review Letters, 88(23), June 2002.

[36] S. Yurgenson, C.-C. Hu, C. Kim, and J. A. Northby. Detachment of metastable helium molecules from helium nanodroplets. European Physical Journal D, 9:153-157, 1999.

[37] C. Hu. Spectroscopy of He 2 floating on the liquid helium surface. Physica B: Condensed Matter, 284-288:107-108, July 2000.

[38] Z. L. Li, N. Bonifaci, F. Aitken, A. Denat, K. Von Haeften, V. M. Atrazhev, and V. A. Shakhatov. Luminescence from liquid helium excited by corona discharges. Dielectrics and Electrical Insulation, IEEE Transactions on, 16(3):742-750, June 2009.

[39] Z. L. Li, N. Bonifaci, F. Aitken, A. Denat, K. von Haeften, V. M. Atrazhev, and V. A. Shakhatov. Spectroscopic investigation of liquid helium excited by a corona discharge: evidence for bubbles and "red satellites". The European Physical Journal Applied Physics, 47(2):22821+, 2009.

[40] Z. L. Li. Décharge couronne dans l'hélium liquide et gaz dense sous champ électrique intense : pré-claquage, transport de charge, spectroscopie d'émission. PhD thesis, University J. Fourier, Grenoble, 2008.

[41] D. N. McKinsey, W. H. Lippincott, J. A. Nikkel, and W. G. Rellergert. Trace detection of metastable helium molecules in superfluid helium by laser-induced fluorescence. Physical Review Letters, 95(11), September 2005. 